\title{
Recuento de los medios electrónicos regionales: ¿Hacia nuevas vertientes de investigación sobre el tema?
}

Claudia Benassini Félix ${ }^{2}$

\section{Introducción}

A

BORDAR EL TEMA de los medios electrónicos regionales suele remitir a ideas concretas y difusas a la vez, a experiencias de exposición y objetos de estudio más o menos delimitados, y a la premisa de que se trata de entidades homogéneas que realizan su quehacer cotidiano de manera similar en cualquier parte del país, aunque bajo diversas modalidades de operación: concesión o permiso comercial, cultural, universitaria o popular. Una premisa falsa, permeada por el etnocentrismo y el desconocimiento de experiencias concretas, producto de condiciones geográficas, económicas, políticas y socioculturales que desembocan en proyectos individuales y colectivos, de alguna manera coyunturales, con semejanzas y diferencias. La imprecisión suele ser producto del desconocimiento de dichas experiencias concretas, tanto porque se generaliza a partir de casos aislados, como por el vacío y la dispersión de información. En suma, el panorama sobre los medios electrónicos regionales está disperso en la geografía nacional, en los archivos y hemerotecas locales, en las publicaciones de circulación amplia o restringida y en los cubículos de los estudiosos del tema - cuando no en su mente, en calidad de proyecto-, que no encuentran la manera de dar salida a su trabajo, o un espacio para la socialización y discusión sobre sus hallazgos e inquietudes.

1. Este trabajo se hizo con el apoyo de la Universidad Iberoamericana, plantel Santa Fe. Participaron Lissete Alvarado, Claudio Flores y Marisela Portillo, quienes se encargaron de la recopilación y sistematización de la información.

2. Universidad Iberoamericana, plantel Santa Fe. 
Esta conclusión no es producto de la especulación, sino del diálogo con quienes tienen inquietudes y se han acercado al estudio de los medios electrónicos desde sus ciudades de origen o de residencia. Las opiniones son diversas y dependen de la experiencia personal. Mientras unos continúan sus esfuerzos con resultados que han culminado en investigaciones concretas y van construyendo su camino, otros han optado por abandonar el tema al menos parcialmente y otros más mantienen vivo su interés, aunque no tienen las condiciones para trabajarlo. Sin embargo, todos coinciden en su importancia y en la necesidad de establecer vínculos para discutir las especificidades de su región y contrastarlas con otras y construir gradualmente el mapa nacional de los medios electrónicos regionales. Estas razones son parte del origen de este trabajo. Paralelamente, Fátima Fernández propuso revivir un proyecto que años antes había iniciado desde el Consejo Nacional para la Enseñanza y la Investigación de las Ciencias de la Comunicación (CONEICC).

La propuesta fue aceptada por los interesados, a quienes se presentó la iniciativa en diversos foros promovidos por el propio Consejo. En Tijuana, durante un taller de investigación de la comunicación, organizado a principios de 1997, se hizo el primer listado de líneas de trabajo sobre medios electrónicos regionales, mismo que se completó en Querétaro y en Veracruz. La diversidad de líneas fue sorprendente y mostró que podía trabajarse de manera secuencial o por separado, en función de la formación académica y los intereses individuales y/o de formación de grupos. Para concretarla fue necesario realizar un diagnóstico bibliohemerográfico sobre medios electrónicos regionales, para detectar el estado de la cuestión y proponer una agenda temática que pudiera desarrollarse bajo diversas modalidades e intereses, individual o colectivamente.

Este trabajo pretende recobrar los esfuerzos emprendidos por el CONEICC y otros investigadores; en un segundo momento, presenta los resultados del diagnóstico de un año de publicaciones sobre el tema. Ambos elementos, aunados a las líneas propuestas por los interesados, apoyaron la elaboración de la agenda temática y las posibles estrategias de organización para trabajos individuales o colectivos y para el intercambio de ideas. Finalmente, el trabajo incluye sugerencias de sitios en Internet a consultar, así como tres directorios anexos que pueden facilitar el contacto con emisoras regionales o con grupos radiofónicos y televisivos con sede en la ciudad de México.

\section{Antecedentes}

Prácticamente desde 1976, año de su creación, el CONEICC ha considerado como uno de sus objetivos prioritarios la investigación sobre medios regionales. Sin 
embargo, hasta septiembre de 1988, bajo la presidencia de Beatriz Solís, Fátima Fernández Christlieb - coordinadora del Comité de Investigación-promovió la realización de un Seminario de Investigación Regional, celebrado en Xalapa. Este seminario derivó en la elaboración de diversos proyectos y productos relacionados con el tema, parte de los cuales se publicó al interior de las instituciones; otra parte ha dado pie a la presentación de ponencias en diversos foros, como los Encuentros de Comunicación periódicamente organizados por el CONEICC. ${ }^{3}$ Simultáneamente, la coordinadora del Seminario estaba inmersa en una investigación sobre el tema que dio como resultado la publicación del libro La radio mexicana: centro y regiones.

En ese momento e incluso desde antes, había además investigadores interesados en el tema como Francisco Aceves, Pablo Arredondo, Gilberto Fregoso y Enrique Sánchez Ruiz, quienes en el entonces Centro de Estudios e Investigación de la Comunicación (CEIC) de la Universidad de Guadalajara se acercaban a los vericuetos de los medios jaliscienses. Estos trabajos inicialmente aparecieron en Comunicación y Sociedad, así como en otras publicaciones de la propia Universidad. Hasta la fecha, en el ahora Departamento de Estudios de la Comunicación Social (DECS) los medios electrónicos regionales, particularmente en Jalisco, son objetos de estudio que se vincula a diversas líneas de investigación.

Por esas mismas fechas, María Antonieta Rebeil publicó en Las redes de Televisa, compilación de Raúl Trejo Delarbre, los resultados preliminares de una investigación sobre el tema. De esa misma época data Espacios del silencio, un conjunto de experiencias de quienes entonces participaban o habían participado en los nacientes sistemas regionales de radio y televisión. ${ }^{4}$ Más o menos en este periodo se ubica también un interés por el estudio de diversas modalidades radiofónicas, particularmente la popular y la indígena. La revista Comunicación y Cultura de la Universidad Autónoma Metropolitana-Xochimilco dedicó un número al tema, con colaboraciones de investigadores latinoamericanos que daban cuenta tanto de experiencias radiofónicas como de estudios y reportes de investigación sobre medios electrónicos regionales. En México, Inés Cornejo, Etelvina Hernández, Felipe Espinoza, Aurora Velasco, Javier Díez de Sollano y

Además se propició el desarrollo y/o conclusión de tesis de licenciatura elaboracias por los propios participantes quienes, aunados a quienes ya estaban titulados y se interesaron en el tema, regresaron a sus centros de trabajo con nuevas estrategias para apoyar a sus alumnos. De hecho, la creación de los Sistemas Regionales de Radio y Televisión constituyó una línea temática importante para la realización de diversos trabajos sobre medios electrónicos regionales. Asimismo, en sus inicios sistemas como el Mexiquense y el Michoacano realizaron seminarios en los que participaron investigadores y productores. En algunos se contó con una memoria escrita y en otros los trabajos aparecieron en publicaciones especializadas $v$ de divulgación. 
Jorge Martínez Lugo, entre otros, realizaron sus tesis de maestría apoyados en trabajo de campo realizado en estaciones radiofónicas populares e indígenas.

Asimismo, desde hace más de diez años la Universidad de Colima también ha dado cabida a la investigación sobre medios electrónicos regionales, a través de una línea del "Programa Cultura". Los resultados de estos trabajos han aparecido en Estudios sobre las Culturas Contemporáneas y en la ya citada Comunicación y Sociedad, entre otras; también la Revista Mexicana de Comunicación ha abierto un espacio al tema de los medios electrónicos regionales. Como parte de estos esfuerzos, también cabe citar los trabajos de Cristina Romo, José Cisneros y Ana María Pippino - centrados en la radio-, los de Ramón Gil Olivo, así como los esfuerzos realizados por académicos e investigadores de diversas instituciones: Manuel Ortiz en la Universidad Autónoma de Baja California, así como Catalina Soto y María Emilia Castillo en la Universidad de Sonora.

Por otra parte, a pesar del interés en abrir nuevos talleres sobre investigación regional - según consta en actas del CONEICC - el tema se retomó a instancias de José Carlos Lozano -entre octubre de 1992 y marzo de 1997 al frente del Comité de Investigación-, quien propuso el proyecto nacional de análisis de contenido comparativo de los medios informativos regionales. Bajo el título tentativo de "Temas, fuentes e imágenes de provincia en los medios informativos mexicanos", la investigación tuvo como objetivo central el análisis del periódico y/o noticiario de televisión de mayor penetración en la ciudad o estado de procedencia de los interesados. El proyecto se inició con un taller de investigación realizado en la Universidad de las Américas-Puebla, en el que participaron representantes de diversas instituciones.

El título final de esta investigación fue Análisis de contenido de temas y fuentes en la información internacional, nacional y local de los principales diarios y noticieros televisivos. Los resultados se publicaron en el Anuario de Investigación de la Comunicación CONEICC $11 .{ }^{5}$ Paralelamente los participantes de este proyecto continuaron sus trabajos, vinculados a sus respectivas instituciones y a las temáticas de su interés. ${ }^{6}$ Otros cambiaron sus líneas de trabajo, a la vez que el auge de los posgrados en comunicación de las Universidad Iberoamericana, plantel Laguna; Universidad Veracruzana y el Tecnológico de Monterrey, cam-

5. El título del artículo es "Temas y fuentes informativas en la prensa mexicana: estudio comparativo". La lista de participantes aparece por orden alfabético: Laura Arellano, Blanca Chong, Alejandra Caña, Mónica Gendreau, Felipe Guicho, Mónica Gutiérrez, José Carlos Lozano, Francisco Javier Martínez, Liliana Morales, Laura Orellana, Manuel Ortiz, Laura Matanzo, Juan Alfonso Priante, Cecilia Quintanilla, Marco Antonio Román y María del Carmen Villarreal.

6. Por ejemplo, la tesis de Maestría en Comunicación de Blanca Chong: Usos sociales de la radio en la región Laguna, de la Universidad Iberoamericana, Plantel Laguna, sustentada en junio de 1998. 
pus Monterrey han impulsado líneas de investigación y tesis sobre los medios electrónicos en sus respectivas ciudades y regiones. Finalmente, como se verá más adelante, las opciones abiertas por Internet constituyen también una importante salida a la investigación sobre medios electrónicos regionales.

\section{Método de trabajo para la sistematización}

Para realizar el diagnóstico sobre el estado de la cuestión, se recopilaron documentos publicados durante 1997 acerca de los medios electrónicos regionales. Estos materiales se sistematizaron con base en tres rubros importantes: los datos generales del documento, el género al que pertenecen y su estructura, es decir, el marco teórico, metodología, hipótesis y conclusiones principales. Inicialmente, se había pensado en que la recopilación y sistematización de estos materiales generaran una base de datos. Sin embargo, debido al escaso número de materiales compilados - y a los datos arrojados por los mismos-, se optó por no realizar esta parte del trabajo y proceder al diagnóstico de los documentos rastreados. De aquí la importancia de los antecedentes sobre la investigación acerca de los medios electrónicos regionales, pues permite contextualizarla en un ámbito de interés más extenso.

Una primera recopilación de información fueron las tesis de licenciatura enviadas por las instituciones afiliadas al CONEICC, a las que se agradece su entusiasmo y colaboración: Escuela de Comunicación Social de Sinaloa, Universidades Autónomas de Baja California, Coahuila, Estado de México, Guerrero, Nuevo León y San Luis Potosí.

También participaron las Universidades de las Américas-Puebla, Colima, del Bajío, Sonora y del Noreste, así como la Escuela de Periodismo Carlos Septién García. Paralelamente se realizó una búsqueda bibliohemerográfica en revistas especializadas como Estudios sobre las culturas contemporáneas, Comunicación y Sociedad, Crítica, revista de la Beremérita Universidad Autónoma de Puebla, y la Revista Mexicana de Comunicación. También se solicitó información a la Cámara Nacional de la Industria de la Radio y la Televisión.

\section{Resultados}

Se localizaron 21 trabajos entre tesis, artículos y avances de investigacińn. F! escaso tamaño de la muestra indica que no es suficiente para hacer un diagnóstico que dé cuenta del estado de la cuestión, mucho menos del interés por la 
investigación sobre los medios electrónicos regionales. ${ }^{7}$ Sin embargo, para evitar reduccionismos, el diagnóstico preliminar se hará en función de los documentos publicados en ese año y partiendo de una retrospectiva.

1. Se observa un marcado interés por el estudio de los emisores, particularmente en las tesis de licenciatura proporcionadas por las instituciones que enviaron trabajos para este reporte. ${ }^{8} \mathrm{~A}$ modo de ejemplo cabe citar Metodología de la producción radiofónica en el grupo FM, de Ana María Carbó, Berenice Cisneros y Guadalupe Díaz Montes, de la Universidad Autónoma de Baja California, Campus Mexicali. También la Escuela de Periodismo Carlos Septién García y las Universidades del Bajío y Autónoma de Guerrero enviaron trabajos representativos de este interés. Asimismo, la Universidad de Sonora ha generado trabajos de este tipo, como producto de un seminario de estudiantes coordinado por María Emilia Castillo, que han derivado en tesis como La radio en Caborca.

2. Las tesis de licenciatura también muestran interés por el análisis de contenido. Es el caso de la Universidad Autónoma del Estado de México, con trabajos como La imagen del candidato priísta a la gubernatura en los periódicos El Sol de Toluca y El Universal (proceso electoral 1993, 2 de febrero al 4 de julio), de Verónica Avilés y Fabiola Cardiel; Análisis de contenido: estudio de caso de la tendencia informativa de El Sol de Toluca y El Heraldo de Toluca en el proceso electoral para presidente de la República en 1994, de Alejandro Macedo García. Esta tendencia también aparece en una tesis de la Universidad del Bajío: Análisis de la estructura y funcionamiento del noticiario televisivo de Radiotelevisión de Guanajuato (canal 4), de Francisco García Muñoz, que conjuga el análisis del emisor y del mensaje.

3. Asimismo, las tesis muestran interés por el uso de los medios electrónicos para fines educativos y/o culturales. Como ejemplo pueden citarse la Propuesta de un programa radiofónico educativo para Ciudad Victoria, Tamaulipas, de María Esther Álvarez Reyes, egresada de la Escuela de Periodismo Carlos Septién García; La problemática de la educación en televisión, de María de Lourdes Domínguez Michelena y Propuesta radiofónica para la educación ecológica, de María del Carmen López y Brígida Ramos, ambas tesis de la Universidad del

7. Además del desarrollo que el tema ha tenido en las Universidades de Colima y Guadalajara, cabe citar los trabajos de Héctor Gómez Vargas de la Universidad Iberoamericana, plantel León, los del equipo que encabeza Ricardo Morales en Tijuana, así como el ya mencionado impulso que ha recibido el tema a raíz del auge de los Posgrados.

8. A pesar del número de instituciones participantes, en este reporte se consignan únicamente las tesis que proporcionaron la información solicitada de acuerdo con los criterios de sistematización $\rightarrow$ que enviaron ejemplares-, incluyendo nombre (s), título del trabajo y universidad de procedencia. Asimismo, cabe destacar que cada institución adoptó sus propios criterios para el envío de las tesis. 
Bajío. Por otra parte, son pocas las tesis de licenciatura que abordan la recepción del mensaje como eje temático. De los casos sistematizados, únicamente dos recurren a encuestas, uno entre televidentes y otro entre radioescuchas.

4. No obstante, el tema de la recepción está presente entre los investigadores. Entre los trabajos sistematizados cabe señalar el de Héctor Gómez Vargas: Biografias radiofónicas: trayectorias y travesías por mundos sociales. ${ }^{9}$ Se trata de un estudio exploratorio, en el que se propone mirar desde los contextos socioculturales donde viven los sujetos, para reconstruir la práctica cultural del consumo radiofónico, específicamente en la ciudad de León. Otros ejemplos son la tesis de maestría de Luis Alfonso Guadarrama, sustentada en $1997,{ }^{10}$ la cual se apoya en los resultados de un protocolo de observación etnográfica realizado entre familias de diversos municipios del Estado de México. Lo mismo cabe señalar sobre el equipo encabezado por Karla Covarrubias de la Universidad de Colima, sobre grupos de discusión en diversas ciudades del país a propósito de la telenovela Mirada de mujer, trabajo iniciado en 1998 y con un buen grado de avance a la fecha. Ambos casos constituyen también ejemplos del empleo de metodologías cualitativas para abordar fenómenos de recepción televisiva. Estos trabajos se suman a los publicados en otros años -incluyendo 1997- por Enrique Sánchez Ruiz, así como las tesis de posgrado ya concluidas o con cierto grado de avance.

5. Buena parte de los trabajos publicados durante 1997 tienen un carácter descriptivo o exploratorio. Esto no debe tomarse como una constante, sino más bien como una característica de los documentos que conformaron la muestra para el análisis, así como el carácter de las publicaciones en que se insertan o su calidad de tesis de licenciatura. En este sentido, salvo el trabajo ya citado de Héctor Gómez Vargas, publicado en una revista especializada, que además forma parte del padrón de publicaciones de excelencia del Consejo Nacional de Ciencia y Tecnología (CONACYT). Las tesis de licenciatura cuentan, en su mayoría, con un marco teórico y con los requisitos propios de cada institución. Sin embargo, abundan los casos en que dichos marcos teóricos son obsoletos; o bien parten de una historia del medio en la ciudad en que se ubican, sin que necesariamente haya una justificación dentro del estudio para la inclusión de un apartado con estas características.

6. Por otro lado, al parecer los medios electrónicos regionales concesionados a particulares sólo se interesan en los estudios de rating y su mercado potencial para la venta de espacios publicitarios. En consecuencia, estos trabajos prácticamente no aportan información valiosa sobre sus receptores. Todo indica que las televisoras y radiodifusoras locales están más preocupadas por vender espacios que por las necesidades y expectativas de sus públicos; además, no tienen un

9. Publicado en Estudios sobre las Culturas Contemporáneas, diciembre de 1997.

10. Familia y televisión: un enfoque sistémico, Universidad Iberoamericana, Santa Fe, 1997. 
perfil definido de los radioescuchas y/o televidentes. En suma, existe un gran desconocimiento sobre el público receptor de los medios electrónicos regionales, así como sobre los entornos socioculturales en los que se insertan dichos públicos.

Asimismo, el hecho de que la información se encuentre dispersa en archivos personales, bibliotecas y hemerotecas dificulta la realización de un diagnóstico más amplio sobre el estado de la cuestión sobre la investigación en materia de medios electrónicos regionales. De aquí las omisiones que pueda tener el mismo estudio. Sin embargo, las observaciones al rigor teórico y metodológico en el abordaje del tema pone en el centro de la discusión el análisis crítico de los estudios, con miras a proponer estrategias para construir los objetos de estudio, así como para señalar ausencias y abandonos. En cuanto a las estrategias metodológicas, particularmente en el caso de las tesis de licenciatura, no hay claridad sobre la toma de decisiones teóricas y en materia de técnicas de investigación. En este sentido, tanto los trabajos que conformaron la muestra, como otros similares, no recurren a ambas perspectivas de forma complementaria, lo que permitiría contar con un panorama más global sobre el fenómeno que se estudia. ${ }^{11}$ En suma, las tesis de licenciatura constituyen un indicador para detectar problemas en materia de teoría e investigación de la comunicación, particularmente en el caso de los medios electrónicos regionales.

En conclusión, durante el periodo estudiado se observa poca investigación sobre los medios electrónicos regionales; o bien, el tema se aborda individualmente por los interesados. En contraste, como ya se había señalado, durante la década de los ochenta, la constitución de los sistemas regionales de radio y televisión impulsó en buena medida la investigación sobre el tema. Hoy vivimos una realidad dicotómica: por un lado, algunos investigadores continúan con su interés por estudiar estos fenómenos desde diversas perspectivas; por otro lado, hay un gran abandono del tema ante las nuevas miradas globalizadoras que dejan de lado los acontecimientos regionales. ${ }^{12}$

A este panorama desde el que suele abordarse el estudio de los medios electrónicos regionales, se une la poca importancia que en muchas instituciones se confiere a la investigación de la comunicación, así como a la falta de organización de proyectos conjuntos que deriven en un mayor conocimiento de la realidad global y de sus especificidades regionales. De aquí la necesidad de

11. De hecho, se observa una tendencia a que ambos componentes de las tesis aparezcan desvinculados uno del otro, sin que haya un hilo conductor que oriente y justifique la toma de decisiones en materia de estrategias teóricas y metodológicas.

12. El tema se ha discutido desde hace varios años en diversos foros nacionales e internacionales. Por un lado se argumenta que la globalización no debe ser entendida sin su contraparte, la regionalización y viceversa. Por otra parte, hay autores que han optado por centrarse en uno de estos polos dados sus intereses y perspectivas desde las cuales construyen su objeto de estudio. 
proponer una agenda temática que seguramente estará incompleta, pero bien puede funcionar como punto de partida para otra más elaborada, de acuerdo con las perspectivas, intereses y características socioculturales de las diversas regiones.

\section{Propuesta de una agenda temática de investigación sobre los medios electrónicos regionales}

El diagnóstico arrojado por el análisis de las publicaciones, aunado al interés del CONEICC y a la revisión de otros trabajos sobre el tema, así como de experiencias de investigación, muestran que el tema de los medios electrónicos regionales está vigente. Sin embargo, son pocos los esfuerzos colectivos que se han emprendido para el desarrollo y cumplimiento de investigaciones sobre el tema. De aquí la propuesta de una agenda que pueda orientar a los interesados, en el entendido de que es un trabajo incompleto: el resto corre por cuenta de individuos, instituciones o grupos de investigadores. Cabe aclarar que algunos de estos temas forman parte de la agenda individual de las universidades ya mencionadas en los primeros apartados de este trabajo, ya sea en calidad de líneas de investigación institucional, o de intereses individuales cuyos esfuerzos se canalizan en su mayoría a la asesoría de tesis.

\section{Hacia una construcción del enfoque regional}

Como se mencionó en el apartado anterior, la investigación vinculada a los medios de comunicación se ha polarizado hacia la globalización o su contraparte, la regionalización. Ciertamente, la decisión depende de los intereses y alcances del trabajo a realizar. Sin embargo, una importante corriente de autores argumenta que, independientemente del enfoque adoptado, ambos extremos son interdependientes, de tal manera que uno no puede comprenderse sin la referencia al otro. Para Anthony Giddens: ${ }^{13}$

la transformación local es parte de la mundialización y de la extensión laboral de las conexiones a través del tiempo y espacio. Así, quien estudie las ciudades actuales en cualquier lugar del mundo, sabe que lo que sucede en un barrio local, seguramente ha sido influenciado por otros factores (...) que operan a una distancia indefinida, lejos del barrio en cuestión. El resultado no es necesariamente, ni siquiera corrientemente, un generalizado conjunto de cambios que actúan en dirección uniforme, al

13. Para una discusión más amplia sobre el tema véase Mowlana y Robertson (1994); Portales (1992 y 1994). 
contrario, en muchas ocasiones, consiste en tendencias mutuamente opuestas (Giddens, 1994:68).

Por su parte, Fátima Fernández Christlieb afirma que la región se presta para ser analizada desde múltiples disciplinas y con objetivos muy distintos. Por lo tanto, el estudioso puede poner la atención en aspectos muy diversos de lo que en ella ocurre:

La región es objeto de estudio de geógrafos, historiadores, sociólogos, promotores culturales, administradores públicos, literatos, economistas y de cuantos quieren profundizar en las diferencias que ofrece la dimensión espacial de los fenómenos sociales. Unos buscan comprender la cuestión regional para aplicar con éxito una medida gubernamental; otros porque trabajan en un centro de investigación dedicado a elaborar catálogos sobre todo lo monografiable; habrá quien se dedique a rastrear las huellas de sus propias raíces; no debe faltar quien se interese por las particularidades locales para expandir un negocio y las razones para caer en este tema pueden ser tan variadas como los ángulos, los enfoques y los matices con que se quiera mirar a las regiones (Fernández Christlieb, 1991:57).

Ambas consideraciones apuntan a que el tamaño de la región es variable; su delimitación depende del objeto de estudio en cuestión, o su extensión frente a lo global. Puede tratarse de una ciudad, un área o un conjunto de países. Incluso la región puede tener distintas delimitaciones geográficas, independientemente de la cercanía, puede haber vínculos en cuanto a toma de decisiones, perfiles socioculturales o multiculturales, como en el caso de algunas ciudades de la frontera norte. Y no se pretende que esta conclusión sea definitiva; más bien busca abrir líneas que puedan orientar un debate conducente a construcciones sobre lo regional, que a su vez sirvan de hilo conductor para determinar criterios de selección en materia de medios electrónicos regionales.

En un segundo momento, este proceso de construcción de lo regional contribuirá a superar el nivel puramente descriptivo ya mencionado en el diagnóstico de las publicaciones. Esta actividad implica también la caracterización del ámbito seleccionado como objeto de estudio desde perspectivas que van de lo geográfico a lo sociocultural y es válida tanto para trabajos individuales como para las comparaciones. De la caracterización regional depende en parte la adecuada contextualización de las siguientes propuestas de trabajo.

\section{La reconstrucción histórica}

Los medios electrónicos regionales han atravesado por procesos históricos similares y diferentes: desde el otorgamiento de concesiones y permisos hasta procesos coyunturales que han propiciado diversas formas de organización. Una 
mirada al directorio de la Cámara Nacional de la Industria de la Radio y Televisión (http://www.cirt.com.mx/) ${ }^{14}$ muestra que los grandes concesionarios de los medios electrónicos poseen intereses en diversos estados del país, por medio de compañías con diferente nombre, aunque los números telefónicos son los mismos y se ubican en la ciudad de México. Hay también grupos con una influencia más regional o local, con intereses vinculados a la prensa. No obstante, cada medio tiene su propia historia, misma que eventualmente puede remitir a un análisis más completo sobre semejanzas y diferencias. Este proceso de reconstrucción exige rigor y criterios metodológicos propios de la etnohistoria, disciplina que forma parte de la antropología sociocultural y que permite la reconstrucción de acontecimientos más recientes y todavía no abordados como objeto de estudio por los métodos utilizados por los historiadores. Según Ángel Palerm:

La etnohistoria podría describirse, sencillamente, como una manera de hacer etnografía utilizando fuentes documentales en lugar del trabajo de campo. Por supuesto, en el plano del trabajo concreto surgen problemas muy complejos, que van desde la crítica de las fuentes y su interpretación, a los análisis semánticos y de contenido. Como indica su nombre, la etnohistoria debe montarse firmemente sobre un adiestramiento profesional de igual competencia en los campos de la etnografía y la historia (Palerm, 1987:35).

En suma, la propuesta de la etnohistoria implica no sólo la consulta de archivos y fuentes de primera mano y bibliohemerográficos, sino también la posibilidad de realizar entrevistas a profundidad, de ser posible, con los participantes en la construcción del proyecto de medios electrónicos en el área seleccionada. Como ya se indicó, parte de este trabajo ya se ha realizado en ciudades como Guadalajara; el ejercicio se está intentando en Veracruz y algunos estados fronterizos, aunque no necesariamente desde la perspectiva señalada. La etnohistoria constituye una opción poco explorada y en muchos casos sustituida por cronologías, que constituyen un buen punto de partida para la realización de este trabajo. En la medida en que individual o colectivamente se reconstruya la etnohistoria de los medios electrónicos regionales, habrá mayores posibilidades de buscar interrelaciones y contextualizarlos en el mapa nacional.

14. En la parte correspondiente a Organización de la Radio y la Televisión se incluyen las emisoras locales que cuentan con página de Internet, además de otros datos, entre los que cabe mencionar las "Veinte razones para afiliarse a la CIRT", misma que puede ser de utilidad no sólo para esta línea temática, sino para otras subsiguientes. 


\section{Las modalidades de organización}

Del punto anterior se desprenden - aunque pueden analizarse por separadolas diversas modalidades de organización de los medios electrónicos regionales. Como se destacó al hablar de la etnohistoria, abundan los casos en que un corporativo de sociedades anónimas posee varias concesiones a lo largo del país. Esto puede derivar en una forma de organización global con diferencias en la ciudad, estado y región de que se trate, a la vez que puede influir en criterios de programación: novedades, repeticiones y la combinación con la oferta local, particularmente noticieros y contenidos dirigidos a públicos específicos. Esta línea también puede aplicarse a emisoras permisionadas y a los sistemas regionales de radio y televisión, aunque con objetivos distintos, vinculados a convenios para intercambio de programación y producciones conjuntas.

La forma de organización también está determinada por el ámbito a través del que circula la programación. En el caso de las emisoras comerciales, la misma señal puede recibirse en diferentes ciudades bajo modalidades que van desde la antena aérea hasta los diversos sistemas de televisión de paga, generalmente a través de cable. Por lo tanto, el análisis de la estructura organizativa debe considerar estas modalidades, incluyendo las compañías que proporcionan los servicios y sus respectivas tarifas.

Esta información proporciona elementos adicionales para detectar estrategias de programación; es decir, los criterios y los públicos a los que se dirige, así como las regiones en las que la programación se transmite vía aérea y aquellas en las que se utilizan sistemas de paga. Las emisoras permisionadas y los sistemas regionales operan bajo lógicas distintas, cuyas estrategias constituyen también una línea de investigación a explorar. Es en este contexto donde comienza a cobrar sentido la reconstrucción del área o áreas seleccionadas como objeto de estudio. Las decisiones en torno a la selección de localidades para instalar emisoras permisionadas y concesionadas, así como la conformación de la programación, se organizan en torno al perfil sociocultural de las audiencias.

Otra veta poco desarrollada dentro de los estudios sobre el emisor es la etnografía de la producción. Se trata de un trabajo que requiere tiempo en horarios definidos, dependiendo del género y el medio seleccionado. Entre los pocos trabajos realizados desde esta perspectiva se encuentran el de Guadalupe Chávez de la Universidad de Colima: El análisis de producción de la telenovela mexicana y su forma moderna y racional de operar ${ }^{15}$ y los de María Elena Hernández de la Universidad de Guadalajara, en la línea de Sociología de la producción de noticias. ${ }^{16}$ Actualmente el Posgrado en Comunicación de la Universidad

15. Tesis de Maestría en Sociología, Universidad Iberoamericana, 1991.

16. Una línea de trabajo que, salvo excepciones, únicamente se ha desarrollado en la UdeG. 
Veracruzana está impulsando también esta línea de trabajo en la realización de tesis. A pesar de las dificultades implícitas en la etnografía de la producción, línea que podría desarrollarse a mediano plazo en el ámbito de los medios electrónicos regionales, ${ }^{17}$ que permitiría establecer comparaciones sobre lógicas de producción entre géneros al interior de emisoras de una ciudad, para posteriormente realizar este trabajo a nivel regional y nacional. Esta propuesta puede aplicarse a radio y televisión en sus diversas modalidades: comercial, cultural y los sistemas regionales.

\section{Circulación y contenido de la programación}

La organización de la programación, aunada a la reconstrucción del contexto regional, proporciona a su vez elementos para analizar sus procesos de circulación a través de los medios electrónicos regionales, sean concesionados o permisionados. Conformado este panorama, puede pasarse a un análisis de contenido de la programación local -incluida la misma que circula en otras ciudades, estados o regiones dispersas a lo largo del país-, así como la destinada a los públicos de estas localidades en particular. Proceder de esta manera supone recurrir en primera instancia a métodos cuantitativos; es decir, al menos construir escalas nominales que den cuenta de los programas, ciudades y estados que reciben la misma programación y sus porcentajes con respecto a la programación local, regional y nacional e incluso antigüedad de la oferta programática y su porcentaje frente a las novedades. Esto conduce a un primer nivel analítico que conforma un panorama de la programación en diversas localidades del país. En un segundo momento, puede pasarse a un análisis de contenido de dicha programación, dependiendo de los objetivos y modelos de procedimiento, aunque se sugieren las herramientas proporcionadas por la semiótica textual, así como a las técnicas proporcionadas por el análisis de contenido.

\section{El contexto sociocultural en el que circula la programación}

Los análisis de la programación, remitidos tanto a las formas de organización de las emisoras - tal como se propuso en los puntos 3 y 4-, permiten llegar a conclusiones relevantes. Sin embargo, los resultados remiten a la investigación -0 al menos a la reflexión- sobre sus implicaciones en el contexto sociocultural

17. El método etnográfico supone un periodo de capacitación previa. Un apoyo importante para formar investigadores en esta línea ha sido el Diplomado itinerante en Métodos y Técnicas de Investigación en Sociedad, Cultura y Comunicación, iniciativa de Jesús Galindo de la Universidad de Colima, actualmente con tres sedes: el Instituto Nacional de Antropología e Historia (INAH), la Universidad Iberoamericana, plantel León y la Universidad Veracruzana. 
en el que están inmersos. Por ejemplo, los noticiarios locales y su papel en procesos políticos previos a las elecciones locales, en sus diversas modalidades, y/o las elecciones presidenciales. Otra propuesta sería la selección de programas por géneros, particularmente los de mayor audiencia en la ciudad, estado y/o región objeto de estudio y sus implicaciones socioculturales en las audiencias específicas (niños, mujeres etc.); puede seleccionarse la oferta programática local o la nacional que se recibe en el área seleccionada.

Es decir, nuevamente la organización y oferta programática de los medios electrónicos regionales es un primer referente. Sin embargo, es recomendable considerar otras variables para la investigación sobre audiencias: la producción de sentido, las características de los canales a través de los que circula la programación, así como sus modalidades de circulación (señal gratuita o de paga, modalidad de la estación permisonada: cultural, indígena, popular, etc.), entre otras opciones. Este acercamiento proporciona un panorama sobre las semejanzas y diferencias entre públicos que reciben un mismo programa. Si es gratuito en unas ciudades y de paga en otras, o incluso si la transmisión es bajo una sola modalidad, el análisis incluye el perfil sociocultural en diversos niveles de complejidad que están presentes desde el inicio de la investigación y se relacionan con los criterios de selección de las regiones seleccionadas, así como con los objetivos que orientan el trabajo, mismos que suelen incluir diversos tipos de comparaciones. ${ }^{18}$

Como en otros puntos de la agenda propuesta, este trabajo puede realizarse de manera independiente, como de hecho sucede en la mayoría de los casos. En este sentido, el empleo de metodologías cualitativas - genealogías, historias de vida, etnografías, grupos de discusión, etc.- aportan información relevante, particularmente para los estudios de caso. Un buen ejemplo es el ya citado trabajo coordinado por Karla Covarrubias de la Universidad de Colima sobre el impacto de la telenovela Mirada de mujer en seis ciudades del país, mediante grupos de discusión. Como ya se indicó en la primera propuesta de la agenda, la reconstrucción de las áreas objeto de estudio aporta elementos para un análisis más profundo de la información recabada, pues las características socioculturales de cada ciudad pueden ser determinantes en la producción de sentido y en la apropiación del género.

18. Por ejemplo, algunos alumnos de Maestría en Comunicación del Tecnológico de Monterrey Campus Monterrey están desarrollando tesis bajo esta línea de investigación. 
Sobre el régimen legal de los medios electrónicos regionales

Como en otros casos, este punto de la agenda no ha sido lo suficientemente abordado por los interesados en el tema. Como ya se indicó, los puntos 2 y 3 de la propuesta, relacionados con la historia y las modalidades de organización de los medios electrónicos regionales, remiten a puntos de partida para enfocar el tema. Sin embargo, una primera reflexión al respecto debe proporcionarla la lectura de leyes y reglamentos en la materia, así como su concreción en el área o región seleccionada para el estudio. Para ello se sugiere la consulta de las páginas www de CONEICC (http://www.coneicc.org.mx); o las páginas Web de la Cámara de Diputados (http://www.camaradediputados.gob.mx/), especialmente el sitio de la Comisión de Radio, Televisión y Cinematografía, mismo que incluye la consulta en línea de la Legislación sobre Medios de Comunicación en México. Ambas páginas www fueron desarrolladas por el Proyecto Internet del Tecnológico de Monterrey, Campus Estado de México. ${ }^{19}$

\section{Estrategias de organización para trabajos colectivos}

En los primeros apartados se destacó como uno de los objetivos que originó la realización de este trabajo la detección del interés por el estudio de los medios electrónicos regionales, para promover la organización y coordinación de proyectos sobre el tema por parte del CONEICC. La propuesta queda abierta al nuevo Comité Coordinador, que podría incluir como parte de su plan de trabajo la realización de talleres sobre investigación regional. Sin embargo, para ello sería indispensable contar con propuestas concretas producto de esta agenda temática -que no agota todas las opciones de investigación-, así como de los intereses de los posibles participantes. Pero eso conlleva riesgos inherentes a las políticas de cada institución, en materia de espacios destinados a la investigación, a la inclusión de todas las temáticas que conforman la agenda individual de los eventuales participantes y, en consecuencia, al compromiso de productos concluidos en fechas acordadas. En suma, el CONEICC puede ser una instancia de

19. Debe reconocerse que hay un gran vacío en la materia, no sólo en lo que se refiere a los medios electrónicos regionales. Los esfuerzos de Beatriz Solís y Ernesto Villanueva por promover diplomados itinerantes entre las instituciones interesadas han contribuido al menos a promover un debate entre académicos y a la inclusión de la cuestión legal en los planes de estudio de licenciatura y posgrado. Sin embargo, hay poca cultura de investigación en la materia, misma que debe promoverse a la luz de los debates en torno a la iniciativa de la Ley Federal de Comunicación Social y sus implicaciones al interior de los medios electrónicos regionales. 
apoyo a un proyecto colectivo con ciertas características. Sin embargo, también es conveniente pensar en otras estrategias de organización:

1. Continúa abierta la posibilidad de seguir individualmente con una agenda de trabajo previamente construida, a la que pueden incorporarse algunas propuestas señaladas en el apartado anterior. Sin embargo, es necesario pensar en mecanismos para la difusión de avances de investigación o de reportes de trabajos concluidos, más allá de órganos institucionales internos de circulación reducida. En este sentido, el Anuario de Investigación de la Comunicación del CONEICC puede ser una alternativa, aunada a la creación de un inventario de publicaciones institucionales en las que aparecen los trabajos sobre medios electrónicos regionales. Dicho inventario puede realizarse de dos maneras complementarias: a través del propio Consejo, que podría incluirlo en la carpeta de trabajo que semestralmente reparte entre los asistentes a las Asambleas y/o subirlo a la página Web (http://www.coneicc.org.mx).

2. El trabajo individual suele enriquecerse por las aportaciones de quienes trabajan temas similares ubicados en distintas partes de la región o del país. La formación de redes de investigadores sobre medios electrónicos regionales cons. tituye una posibilidad ya explorada - aunque no estrictamente sobre el tema-, que propicia el intercambio de opiniones de diversa índole: desde sugerencias bibliohemerográficas y dónde conseguirlas, hasta opiniones sobre estrategias metodológicas y comentarios que pueden o no incorporarse a la investigación. Un ejemplo interesante se encuentra en la Red de Investigación de la Comunicación Compleja (RICC), creada a iniciativa de Jesús Galindo y Octavio Islas, que, como señala en su página Web (http://www.mexicocomunica.org.mx/),

es una forma de red sistémica en proceso de configuración. Su integración se establece con base a los vínculos que se desarrollan entre grupos de trabajo e individuos asociados. Los grupos que conforman la Red de Comunicación Compleja pueden ser de varios tipos: institucionales, de interés y temáticos. Los grupos institucionales residen en universidades o bien, se trata de aparatos académicos. Los grupos de interés, son los que se forman por un asunto emergente como un proyecto de investigación o alguna otra actividad única y de tiempo determinado. Los grupos temáticos son los que se forman por afinidad en algún asunto, por individuos u otros grupos, en forma más o menos permanente y desde distintos lugares institucionales o geográficos.

Asimismo, la Red de Comunicación Compleja ofrece varias formas de flujo constructivo como sistema de trabajo: Metodología de acción, desde sus áreas constitutivas elementales, el trabajo, la investigación y la creación; productos terminados en investigación y creación; y cultura de investigación, cultura de información y cultura de comunicación, como espacio de acción, como servicio o asesoría, y como sistema de vínculos. La RICC está en posibilidades de abrir un 
foro de discusión sobre medios electrónicos regionales e incluso de registrar una investigación sobre el tema a la que podrían vincularse los interesados vía consulta de la página y correo electrónico. Aun cuando la limitante es que quienes no cuentan con recursos tecnológicos para acceder a Internet, es cada vez mayor el número de usuarios que cuentan con el equipo de manera personal y/o institucional. De esta forma, el trabajo individual se socializa, a la vez que puede cristalizar en proyectos conjuntos realizados por investigadores ubicados en diversos puntos del país.

A modo de ejemplo, el 18 de agosto de 1998 la RICC abrió un foro de discusión sobre la telenovela Mirada de mujer, a sugerencia del equipo de investigadores que, bajo la coordinación de Karla Covarrubias, organizaron los grupos de discusión en sus respectivas ciudades. El foro da cuenta de opiniones sobre el proyecto y su metodología, así como sobre la telenovela. En suma, se trata de un espacio de encuentro y diálogo virtual entre investigadores, colegas y televidentes.

Cuando este reporte comience a circular, la investigación sobre medios electrónicos regionales contará ya con su espacio en la RICC, en el que se registrarán las líneas de investigación propuestas en este documento, aunadas a otras de interés por parte de quienes se incorporen a este primer intento de organización colectiva; también habrá un foro de discusión sobre el tema. Un siguiente paso, que podrá resolverse de manera conjunta entre los interesados, será la forma de socializar material bibliohemerográfico, tanto el sugerido en la última parte de este reporte, como el que vayan proponiendo los visitantes a este espacio.

3. Por último, es importante subrayar los esfuerzos de instituciones como el Programa Cultura de la Universidad de Colima por realizar investigaciones colectivas en diferentes ciudades del país a propósito de asuntos de relevancia regional y nacional. Otro caso es el de José Carlos Lozano, quien a raíz de su investigación sobre Análisis de contenido de temas y fuentes en la información internacional, nacional y local de los principales diarios y noticieros televisivos, emprendió un proyecto similar entre investigadores latinoamericanos vía correo electrónico.

En suma, aprovechar las posibilidades abiertas por la red de redes comienza a volverse una herramienta de uso cotidiano para investigaciones colectivas. Una oportunidad que debe aprovecharse en el caso de los medios electrónicos regionales para unir esfuerzos y poner en contacto a quienes han realizado su trabajo de manera aislada. 


\section{Conclusiones}

Aun cuando el diagnóstico bibliohemerográfico muestra que durante 1997 fue escasa la producción sobre medios electrónicos regionales, el sondeo efectuado durante ese mismo año entre posibles interesados en el tema muestra que el tema continúa vigente. De aquí la importancia de proponer esta agenda, contextualiżada no sólo en un año de producción localizada entre quienes hicieron llegar documentos para su análisis, entre quienes han venido desarrollando el tema y entre quienes mostraron interés por iniciarlo bajo una o varias líneas de investigación. Como pude observarse, para este ejercicio de contextualización y de propuesta, los antecedentes también juegan un papel importante. Para llevar a cabo dentro de estas líneas temáticas, sea como iniciado o como quien lo viene haciendo cotidianamente, se sugieren algunas estrategias de organización para trabajos individuales o colectivos.

Como ya se indicó en su momento, la agenda está incompleta; llenar los huecos es una tarea que corresponde a todos y cada uno de los interesados en el tema. Por lo tanto, un primer ejercicio es la discusión sobre su viabilidad individual, institucional a interinstitucional, para posteriormente completarla con las líneas temáticas no incluidas en este reporte, pero que han sido abordadas por los estudiosos de un tema que, por diversas razones, concentra y dispersa. Por el momento, en la mayoría de los casos esta dispersión ha propiciado la atomización de esfuerzos. Es deseable que esta propuesta, aunada al interés, contribuya más a la concentración y a un mayor conocimiento sobre las especificidades de los medios electrónicos regionales. En este sentido, es ilustrativa la afirmación de Jorge González y Guadalupe Chávez sobre la importancia de asumir:

...en cada ciudad y en cada región el reto de generar los equipos capaces de producir su propia información sobre los procesos culturales que les afectan, el reto de capacitarse para no sólo poder generar los datos, sino también proveerse de las herramientas y los cuestionamientos pertinentes para interpretarlos, para dotar las cifras de sentido. Con ello, tendremos al menos la posibilidad de que nuestra forzada entrada al mundo de la globalización económica y de la mundialización de las formas culturales, pueda ser más participativa y más expansiva (González y Chávez, 1996:69). 


\section{Bibliografía}

Fernández Christlieb, Fátima (1991): La radio mexicana: centro y regiones, Editorial Juan Pablos, México.

Giddens, Anthony (1994): Consecuencias de la modernidad, Alianza Editorial, Madrid.

González, Jorge y Chávez, María Guadalupe (1996): La cultura en México, I: cifras clave, Conaculta-Universidad de Colima, México.

Mowlana, Hamid (1994): "Shapes of the future: International Communications in the Twenty-first Century", en The Journal of International Communication, volumen 1, junio de 1994, Universidad de Sidney, Australia.

Palerm, Ángel (1987): Teoría etnológica, Universidad Autónoma de Querétaro, México.

Portales, Diego (1992): “La integración televisiva: desde lo global hasta lo local", en La integración cultural latinoamericana. Tomo III de las memorias del viI Encuentro Latinoamericano de Facultades de Comunicación Social, Acapulco, Guerrero, México.

- (1994): Utopías en el mercado. Las Producciones del Ornitorrinco, Santiago de Chile. 